\title{
\begin{tabular}{l|l} 
Mibraries & DSpace@MIT
\end{tabular}
}

\author{
MIT Open Access Articles
}

\section{RADIO EMISSION FROM SN 1994I IN NGC 5194 (M 51): THE BEST-STUDIED TYPE Ib/c RADIO SUPERNOVA}

The MIT Faculty has made this article openly available. Please share how this access benefits you. Your story matters.

Citation: Weiler, Kurt W., Nino Panagia, Christopher Stockdale, Michael Rupen, Richard A. Sramek, and Christopher L. Williams. "RADIO EMISSION FROM SN 1994I IN NGC 5194 (M 51): THE BEST-STUDIED TYPE Ib/c RADIO SUPERNOVA." The Astrophysical Journal 740, no. 2 (October 3, 2011): 79. (C) 2011 The American Astronomical Society

As Published: http://dx.doi.org/10.1088/0004-637x/740/2/79

Publisher: IOP Publishing

Persistent URL: http://hdl.handle.net/1721.1/95632

Version: Final published version: final published article, as it appeared in a journal, conference proceedings, or other formally published context

Terms of Use: Article is made available in accordance with the publisher's policy and may be subject to US copyright law. Please refer to the publisher's site for terms of use. 


\title{
RADIO EMISSION FROM SN 1994I IN NGC 5194 (M 51): THE BEST-STUDIED TYPE Ib/c RADIO SUPERNOVA
}

\author{
Kurt W. Weiler ${ }^{1}$, Nino Panagia ${ }^{2,6}$, Christopher Stockdale ${ }^{3}$, \\ Michael Rupen ${ }^{4}$, Richard A. SRAmeK ${ }^{4}$, AND Christopher L. Williams ${ }^{5}$ \\ ${ }^{1}$ Naval Research Laboratory, Code 7213, Washington, DC 20375-5320, USA; Kurt.Weiler@nrl.navy.mil \\ ${ }^{2}$ Space Telescope Science Institute, Baltimore, MD 21218, USA; panagia@ stsci.edu \\ ${ }^{3}$ Physics Department, Marquette University, Milwaukee, WI 53201-1881, USA; Christopher.Stockdale @ marquette.edu \\ ${ }^{4}$ National Radio Astronomy Observatory, Socorro, NM 87801, USA; mrupen@nrao.edu, dsramek@nrao.edu \\ ${ }^{5}$ MIT Kavli Institute for Astrophysics and Space Research, Cambridge, MA 02139, USA; clmw@ mit.edu \\ Received 2011 May 17; accepted 2011 July 20; published 2011 October 3
}

\begin{abstract}
We present the results of detailed monitoring of the radio emission from the Type Ic supernova SN 1994I from three days after optical discovery on 1994 March 31 until eight years later at age 2927 days on 2002 April 5. The data were mainly obtained using the Very Large Array at the five wavelengths of $\lambda \lambda 1.3,2.0,3.6,6.2$, and $21 \mathrm{~cm}$ and from the Cambridge $5 \mathrm{~km}$ Ryle Telescope at $\lambda 2.0 \mathrm{~cm}$. Two additional measurements were obtained at millimeter wavelengths. This data set represents the most complete, multifrequency radio observations ever obtained for a Type Ib/c supernova. The radio emission evolves regularly in both time and frequency and is well described by established supernova emission/absorption models. It is the first radio supernova with sufficient data to show that it is clearly dominated by the effects of synchrotron self-absorption at early times.
\end{abstract}

Key words: galaxies: individual (NGC 5194 [M 51]) - radio continuum: stars - stars: mass-loss - supernovae: general - supernovae: individual (SN 1994I)

Online-only material: color figure

\section{INTRODUCTION}

\subsection{Background}

By 1941 Minkowski (1941) had recognized at least two classes of supernovae ( $\mathrm{SNe}$ )-Type I with no hydrogen in their optical spectra and Type II with hydrogen lines in their optical spectra. By 1980 radio observations of supernovae began to play a role with the clear detection of SN 1979C (Weiler \& Sramek 1980; there had been only one earlier detected radio supernova (RSN), SN 1970G, by Gottesman et al. 1972 and Goss et al. 1973 but the Westerbork telescope was unable to cleanly separate its radio emission from a nearby $\mathrm{H}$ II region) and the monitoring of its radio light curves at multiple radio frequencies for many years thereafter (Montes et al. 2000). The radio detection and monitoring of SN 1980K quickly followed (Weiler et al. 1986; Montes et al. 1998). However, both objects were optically identified as Type II SNe. At that time, little was known about the radio emission from $\mathrm{SNe}$ and the statistics with only two objects were very poor. However, the radio detection of SN 1983N (Sramek et al. 1984, also labeled SN 1983.51 by those authors), SN 1984L (Panagia et al. 1986), and SN 1990B (van Dyk et al. 1993) appeared to establish radio detections of Type I SNe. Around that time, Panagia et al. (1986) proposed that, from their optical spectra, Type I SNe were not a homogeneous class but split into at least two subclasses. The appearance of SN 1994I, the subject of this paper, finally yielded a bright, nearby object for detailed study of a "radio-loud" Type I supernova.

Currently, our understanding of the classes of $\mathrm{SNe}$ is such that the category of Type I supernovae is clearly bifurcated. One now speaks of Type Ia SNe (which still have never been

\footnotetext{
6 Also at INAF-CT Osservatorio Astrofisico di Catania, Via S. Sofia 79, I-95123 Catania, Italy; Supernova Ltd., OYV \#131, Virgin Gorda, British Virgin Islands.
}

detected in the radio; see Panagia et al. 2006) as originating in the deflagration of small stars, such as accreting or merging white dwarfs or black holes, and Type $\mathrm{Ib} / \mathrm{c}$ and Type II SNe (of which there are also numerous subclasses) as originating in the core collapse of massive stars with a zero-age main sequence masses of $>8 M_{\odot}$.

Particularly since the discovery of an association of SN 1998bw with GRB 980425 (Sadler et al. 1998), the study of Type $\mathrm{Ib} / \mathrm{c} \mathrm{SNe}$ has greatly increased (as explained below, the optical spectroscopic differences between Type Ib and Type Ic $\mathrm{SNe}$ are so slight that we treat them as a single class). However, further work has found that most Type $\mathrm{Ib} / \mathrm{c} \mathrm{SNe}(\geqslant 97 \%)$ are not associated with detectable gamma-ray bursts (GRBs; Berger et al. 2003a; Soderberg et al. 2006).

Even with the enhanced study of Type $\mathrm{Ib} / \mathrm{c} \mathrm{SNe}$ since SN 1998bw, most are still relatively poorly sampled in the radio (see, e.g., Berger et al. 2003b; Soderberg et al. 2004, 2005, 2010) and, even at the present time, no Type Ib/c SN has been as thoroughly studied in the radio like SN 1994I presented in this paper.

\subsection{SN $1994 I$}

SN 1994I in M 51 (NGC 5194) was discovered at magnitude $V=13$ m 5 on 1994 April 2.17 UT (Puckett \& Armstrong 1994) with independent discoveries by other observers following quickly (see IAUC 5961). Richmond \& Filippenko (1994) confirmed the discovery as an SN and noted it as being "very blue." Armus \& Mazzarella (1994) obtained moderatedispersion spectra and noted "broad undulations superimposed on a generally featureless continuum."

Initial confusion arose as to the type identification of the SN with Schmidt \& Kirshner (1994) first (incorrectly) identifying it as a Type II SN based on unreduced spectra taken by J. Peters and G. Bernstein during 1994 April 3.37-3.52 UT. The spectra, however, did suggest the SN location in NGC 5194 
(M51) by identifying strong $\mathrm{Na} \mathrm{D}$ lines at the recession velocity of the galaxy. By 1994 April 4.4 UT, Filippenko et al. (1994) suggested from new spectra that SN 1994I was a Type Ib SN. However, they noted the "possible presence of weak $\mathrm{H} \alpha$ " and suggested that, although the presence of hydrogen would "technically make it a Type IIb," the steep drop of the continuum toward the blue and near-ultraviolet was more consistent with a Type Ib identification, as were other aspects of the spectrum. Finally, Schmidt et al. (1994) confirmed that SN 1994I was not a Type II SN and Phillips (1994) pointed out the spectral similarities to the Type Ic SN 1983V. The Type Ic identification was also confirmed by Clocchiatti et al. (1994). (Note: because the spectral differences between the SN Type Ib and Type Ic optical classes are slight-Type Ib show strong He I absorption while Type Ic show weaker He I absorption-and there are no obvious radio differences, we shall hereafter refer to Type $\mathrm{Ib} / \mathrm{c}$ SNe as one class.) Richmond (1994) discovered a red star near the SN position on Hubble Space Telescope (HST) Planetary Camera images from 1992 which he suggested as a possible progenitor to the $\mathrm{SN}$, although his suggestion was disputed by Kirshner (1994).

On 1994 April 3.17 UT, Rupen et al. (1994) detected radio emission at the position of SN 1994I with the Very Large Array $(\mathrm{VLA})^{7}$ at both $\lambda 1.3 \mathrm{~cm}(22.4 \mathrm{GHz})$ and $\lambda 3.6 \mathrm{~cm}(8.4 \mathrm{GHz})$. The radio observations established an accurate position of R.A. $(\mathrm{J} 2000)=13^{\mathrm{h}} 29^{\mathrm{m}} 54^{\mathrm{s}} .12$, decl. $(\mathrm{J} 2000)=+47^{\circ} 11^{\prime} 30^{\prime \prime} .4$ with an uncertainty of $\pm 0^{\prime \prime} .1$ in each coordinate with an offset from the radio nucleus of M 51 of $14^{\prime \prime} .3$ east and 12". 3 south. Morrison \& Argyle (1994) report a slightly different optical astrometric position of R.A.(J2000) $=13^{\mathrm{h}} 29^{\mathrm{m}} 54^{\mathrm{s}} .072$, decl.(J2000) $=$ $+47^{\circ} 11^{\prime} 30^{\prime}$. 50 with uncertainty of $\pm 00^{\prime}$. 08 in each coordinate, a difference of $\sim 0^{\prime \prime} .5$ in R.A. and $\sim 0^{\prime} .1$ in decl. This optical/radio position difference might imply that there is a significant offset between the centroids of the optical and radio emission (unlikely since, at the distance of M51 at $8.9 \mathrm{Mpc}$; 0.5 is $21.5 \mathrm{pc}$ ) or, more likely, that there is an offset between the radio reference frame and the FK5-based optical reference frame in that part of the sky. Tully (1988) determined a distance of 7.7 Mpc (assuming $H_{0}=75 \mathrm{~km} \mathrm{~s}^{-1} \mathrm{Mpc}^{-1}$ ) to NGC 5194. Accepting the Tully (1988) method and using $H_{0}=65 \mathrm{~km} \mathrm{~s}^{-1} \mathrm{Mpc}^{-1}$, we will adopt $8.9 \mathrm{Mpc}$ as the distance to SN 1994I. Other distances to M51 range from 9.6 Mpc $\left(H_{0}=57\right.$; derived from $\mathrm{H}$ II regions and brightest stars by Sandage \& Tammann 1974) to $8.4 \mathrm{Mpc}$ (Feldmeier et al. 1997) from planetary nebula luminosity.

Ultraviolet measurements were reported from the International Ultraviolet Explorer on 1994 April 3.35, 4.9, and 5.13 by Rodriguez-Pascual et al. (1994) and an infrared detection was reported on 1994 April 23.2 UT by Rudy \& Dotan (1994) in the $J, H$, and 2.00-2.32 $\mu \mathrm{m}$ windows. An unabsorbed soft $\mathrm{X}$-ray luminosity upper limit of $<1.5 \times 10^{38} \mathrm{erg} \mathrm{s}^{-1}$ for a distance to M51 of 7.7 Mpc was reported by Lewin et al. (1994) from the ROSAT satellite for 1994 May 22 and subsequent Xray studies yielded the detection of SN 1994I with ROSAT at 82 days after explosion with $(1.82 \pm 4.2) \times 10^{38} \mathrm{erg} \mathrm{s}^{-1}$ (Immler et al. 1998). The Chandra X-ray Facility at 2271 and 2639 days after explosion reported $(1.4 \pm 0.7) \times 10^{37} \mathrm{erg} \mathrm{s}^{-1}$ and $(1.2 \pm 0.5) \times 10^{37} \mathrm{erg} \mathrm{s}^{-1}$, respectively (Immler et al. 2002). Immler et al. (2002) determined a best-fit X-ray rate of decline proportional to $t^{-1}$ and a circumstellar medium (CSM) density profile of $\rho_{\mathrm{CSM}} \propto r^{-1.9 \pm 0.1}$, consistent at a constant

\footnotetext{
7 The VLA telescope of the National Radio Astronomy Observatory is operated by Associated Universities, Inc. under a cooperative agreement with the National Science Foundation.
}

mass-loss rate/constant wind velocity for the $\mathrm{SN}$ progenitor which would yield a CSM density profile of $\rho_{\mathrm{CSM}} \propto r^{-2}$.

Prediscovery limits are also available. Richmond et al. (1994) report that SN 1994I was not present to a limiting $R$ magnitude of $\sim 16.2$ on 1994 March 28 UT and a prediscovery measurement with the VLA by Tongue et al. (1994) found a $3 \sigma$ upper limit for $\lambda 21 \mathrm{~cm}$ emission from that position of $<2.3 \mathrm{mJy}$ on 1994 February 12.

Due to its proximity $(8.9 \mathrm{Mpc}$; Tully 1988 recalculated for $H_{0}=65 \mathrm{~km} \mathrm{~s}^{-1} \mathrm{Mpc}^{-1}$ ) and the fact that such an early radio detection was obtained, we quickly started observations at all available wavelengths with the VLA. Regular monitoring of SN 1994I with the VLA at $\lambda \lambda 1.3 \mathrm{~cm}(22.5 \mathrm{GHz}), 2 \mathrm{~cm}$ (14.9 GHz), $3.6 \mathrm{~cm}(8.4 \mathrm{GHz}), 6 \mathrm{~cm}(4.9 \mathrm{GHz})$, and $20 \mathrm{~cm}$ $(1.4 \mathrm{GHz})$ then continued until the end of 1994 with sporadic measurements thereafter. G. G. Pooley \& D. A. Green (1994, private communication) also monitored the SN extensively at $2 \mathrm{~cm}(15.2 \mathrm{GHz})$ with the Ryle Telescope. These data are listed in Table 1. Two additional observations were taken with the Caltech Owens Valley Radio Observatory (OVRO) millimeter interferometer at $3 \mathrm{~mm}(99 \mathrm{GHz})$ and at $1.4 \mathrm{~mm}(218 \mathrm{GHz}$; Phillips et al. 1994). These are listed in Table 2.

\section{RADIO OBSERVATIONS}

\subsection{Flux Density Measurements}

The VLA radio observations reported here were made between 1994 April 3 and 2002 April 5. VLA phase and flux density calibration and data reduction followed standard procedures (see, e.g., Weiler et al. 1986, 1990), using 3C 286 as the primary flux density calibrator and $\mathrm{J} 1418+546$ as the secondary flux density and phase calibrator. As noted in the footnotes to Table 3, two measurements on 1994 April 3 used the calibrator $\mathrm{J} 1415+463$ and one measurement on 2002 April 5 used the calibrator $\mathrm{J} 1327+434$. Table 3 lists the measured or interpolated (or extrapolated) flux densities of the secondary calibrators. For the phase calibration, $\mathrm{J} 1327+434, \mathrm{~J} 1415+463$, and $\mathrm{J} 1418+546$ have defined positions as listed in the NRAO VLA calibrator catalog.

The flux density measurements for SN 1994I are listed in Tables 1 and 2 and plotted in Figure 1 with the filled squares representing actual detections and inverted open triangles representing $3 \sigma$ upper limits. Also shown in Figure 1 are the best-fit model curves discussed below in Sections 3 and 4 with the set of parameters reported in Table 4.

The flux density measurement error given for the VLA measurements in Table 1 is a combination of the rms map error, which measures the contribution of small unresolved fluctuations in the background emission and random map fluctuations due to receiver noise, and a basic fractional error $\epsilon$ included to account for the normal inaccuracy of VLA flux density calibration and possible deviations of the primary calibrator from an absolute flux density scale. These final errors $\left(\sigma_{f}\right)$ are taken as

$$
\sigma_{f}^{2}=\left(\epsilon S_{0}\right)^{2}+\sigma_{0}^{2},
$$

where $S_{0}$ is the measured flux density, $\sigma_{0}$ is the map rms for each observation, and $\epsilon=0.10$ for $20 \mathrm{~cm}, \epsilon=0.05$ for 6 and $3.6 \mathrm{~cm}, \epsilon=0.075$ for $2 \mathrm{~cm}$, and $\epsilon=0.10$ for $1.2 \mathrm{~cm}$. For all of the Cambridge measurements at $2 \mathrm{~cm}, \epsilon=0.10$ was assumed.

\subsection{Spectral Indices}

With such extensive data, we can also calculate the spectral index evolution for SN 1994I and these are shown in Figure 2 
Table 1

Centimeter Flux Density Measurements for SN 1994I

\begin{tabular}{|c|c|c|c|c|c|c|c|}
\hline $\begin{array}{l}\text { Obs. } \\
\text { Date }\end{array}$ & $\begin{array}{l}\text { Age } \\
\text { (days) }\end{array}$ & $\begin{array}{l}\text { Tel. } \\
\text { Config. }\end{array}$ & $\begin{array}{c}S(20 \mathrm{~cm}) \pm \mathrm{rms} \\
(\mathrm{mJy})\end{array}$ & $\begin{array}{c}S(6 \mathrm{~cm}) \pm \mathrm{rms} \\
(\mathrm{mJy})\end{array}$ & $\begin{array}{c}S(3.6 \mathrm{~cm}) \pm \mathrm{rms} \\
(\mathrm{mJy})\end{array}$ & $\begin{array}{c}S(2 \mathrm{~cm}) \pm \mathrm{rms} \\
(\mathrm{mJy})\end{array}$ & $\begin{array}{c}S(1.3 \mathrm{~cm}) \pm \mathrm{rms} \\
(\mathrm{mJy})\end{array}$ \\
\hline 1994 Mar 31 & $=0.00$ & & & & & & \\
\hline 1994 Apr 3 & 3.167 & VLA-A & & & $0.554 \pm 0.062$ & & $2.580 \pm 0.388$ \\
\hline 1994 Apr 4 & 4.120 & VLA-A & & & $0.737 \pm 0.120$ & $2.780 \pm 0.469$ & $3.707 \pm 0.891$ \\
\hline 1994 Apr 5 & 5.350 & VLA-A & $<0.090^{\mathrm{a}}$ & $0.477 \pm 0.091$ & $1.743 \pm 0.108$ & $6.300 \pm 0.522$ & $8.299 \pm 0.891$ \\
\hline 1994 Apr 6 & 6.040 & VLA-A & & $0.700 \pm 0.115$ & & & \\
\hline 1994 Apr 6 & 6.135 & Camb & & & & $10.800 \pm 1.472$ & \\
\hline 1994 Apr 6 & 6.483 & VLA-A & & $1.400 \pm 0.115$ & & & \\
\hline 1994 Apr 7 & 7.028 & VLA-A & & & $2.412 \pm 0.152$ & & \\
\hline 1994 Apr 7 & 7.070 & VLA-A & & & $3.295 \pm 0.190$ & & \\
\hline 1994 Apr 7 & 7.190 & Camb & & & & $13.000 \pm 2.385$ & \\
\hline 1994 Apr 7 & 7.462 & VLA-A & & & $3.656 \pm 0.194$ & $13.100 \pm 1.062$ & $16.300 \pm 1.676$ \\
\hline 1994 Apr 8 & 8.020 & Camb & & & & $14.500 \pm 2.086$ & \\
\hline 1994 Apr 8 & 8.288 & VLA-A & $<0.207^{\mathrm{a}}$ & $1.250 \pm 0.153$ & $4.990 \pm 0.250$ & $14.698 \pm 1.202$ & $15.458 \pm 1.673$ \\
\hline 1994 Apr 9 & 9.180 & Camb & & & & $16.000 \pm 2.561$ & \\
\hline 1994 Apr 9 & 9.386 & VLA-A & & $2.020 \pm 0.144$ & $6.514 \pm 0.334$ & $17.200 \pm 1.409$ & $17.100 \pm 1.810$ \\
\hline 1994 Apr 10 & 10.561 & VLA-A & $<0.225^{\mathrm{a}}$ & $2.123 \pm 0.176$ & $8.930 \pm 0.455$ & $19.171 \pm 1.488$ & $18.593 \pm 2.028$ \\
\hline 1994 Apr 11 & 11.180 & Camb & & & & $17.500 \pm 2.658$ & \\
\hline 1994 Apr 12 & 12.180 & Camb & & & & $17.200 \pm 2.282$ & \\
\hline 1994 Apr 12 & 12.395 & VLA-A & $<0.132^{\mathrm{a}}$ & $4.120 \pm 0.221$ & $11.43 \pm 0.577$ & $15.860 \pm 1.249$ & $12.400 \pm 1.349$ \\
\hline 1994 Apr 13 & 13.220 & Camb & & & & $13.900 \pm 2.436$ & \\
\hline 1994 Apr 13 & 13.372 & VLA-A & $<0.162^{\mathrm{a}}$ & $4.643 \pm 0.271$ & $12.605 \pm 0.646$ & $17.178 \pm 1.365$ & $12.610 \pm 1.414$ \\
\hline 1994 Apr 14 & 14.220 & Camb & & & & $15.600 \pm 2.536$ & \\
\hline 1994 Apr 14 & 14.367 & VLA-A & $0.300 \pm 0.067$ & $5.053 \pm 0.267$ & $13.000 \pm 0.660$ & $16.500 \pm 1.382$ & \\
\hline 1994 Apr 15 & 15.220 & Camb & & & & $15.000 \pm 2.500$ & \\
\hline 1994 Apr 15 & 15.360 & VLA-A & $<0.258^{\mathrm{a}}$ & $5.587 \pm 0.292$ & $14.500 \pm 0.733$ & $16.500 \pm 1.364$ & $10.500 \pm 1.178$ \\
\hline 1994 Apr 16 & 16.220 & $\mathrm{Camb}$ & & & & $16.500 \pm 2.593$ & \\
\hline 1994 Apr 16 & 16.356 & VLA-A & $0.370 \pm 0.095$ & $6.451 \pm 0.338$ & $16.200 \pm 0.820$ & $15.600 \pm 1.236$ & $11.000 \pm 1.252$ \\
\hline 1994 Apr 17 & 17.210 & Camb & & & & $16.100 \pm 2.200$ & \\
\hline 1994 Apr 17 & 17.363 & VLA-A & $0.463 \pm 0.105$ & $7.664 \pm 0.400$ & $17.200 \pm 0.871$ & $16.900 \pm 1.328$ & $10.600 \pm 1.185$ \\
\hline 1994 Apr 18 & 18.199 & VLA-A & & $7.390 \pm 0.392$ & $15.770 \pm 0.810$ & $15.743 \pm 1.215$ & $10.590 \pm 1.205$ \\
\hline 1994 Apr 18 & 18.210 & Camb & & & & $15.900 \pm 2.555$ & \\
\hline 1994 Apr 19 & 19.220 & Camb & & & & $14.000 \pm 2.441$ & \\
\hline 1994 Apr 20 & 20.200 & $\mathrm{Camb}$ & & & & $13.500 \pm 2.018$ & \\
\hline 1994 Apr 20 & 20.494 & VLA-A & $0.421 \pm 0.107$ & $9.250 \pm 0.493$ & $16.483 \pm 0.850$ & $8.757 \pm 0.960$ & $5.673 \pm 1.185$ \\
\hline 1994 Apr 21 & 21.200 & Camb & & & & $14.000 \pm 2.052$ & \\
\hline 1994 Apr 22 & 22.210 & Camb & & & & $13.000 \pm 1.985$ & \\
\hline 1994 Apr 23 & 23.056 & VLA-A & $0.386 \pm 0.089$ & & & & \\
\hline 1994 Apr 23 & 23.150 & Camb & & & & $12.000 \pm 1.921$ & \\
\hline 1994 Apr 24 & 24.336 & VLA-A & $0.688 \pm 0.125$ & $11.130 \pm 0.568$ & & & \\
\hline 1994 Apr 25 & 25.140 & Camb & & & & $10.000 \pm 1.414$ & \\
\hline 1994 Apr 25 & 25.333 & VLA-A & $0.720 \pm 0.122$ & $10.760 \pm 0.551$ & $12.300 \pm 0.651$ & & $4.620 \pm 0.816$ \\
\hline 1994 Apr 28 & 28.163 & VLA-A & $0.683 \pm 0.115$ & $13.713 \pm 0.693$ & $14.723 \pm 0.760$ & $7.503 \pm 0.733$ & $4.990 \pm 0.706$ \\
\hline 1994 Apr 29 & 29.200 & Camb & & & & $10.000 \pm 1.281$ & \\
\hline 1994 Apr 30 & 30.320 & VLA-A & $1.618 \pm 0.167$ & $14.063 \pm 0.726$ & $14.650 \pm 0.757$ & $8.720 \pm 0.848$ & $6.293 \pm 0.971$ \\
\hline 1994 May 2 & 32.140 & Camb & & & & $9.800 \pm 1.400$ & \\
\hline 1994 May 3 & 33.130 & Camb & & & & $9.100 \pm 1.212$ & \\
\hline 1994 May 3 & 33.279 & VLA-BnA & $1.170 \pm 0.142$ & $16.823 \pm 0.847$ & $11.440 \pm 0.591$ & & $3.988 \pm 0.624$ \\
\hline 1994 May 10 & 40.228 & VLA-BnA & $2.660 \pm 0.267$ & $17.550 \pm 0.880$ & $13.040 \pm 0.656$ & $5.330 \pm 0.477$ & $2.180 \pm 0.555$ \\
\hline 1994 May 12 & 42.090 & Camb & & & & $8.000 \pm 1.281$ & \\
\hline 1994 May 15 & 45.179 & VLA-BnA & $2.470 \pm 0.270$ & $16.995 \pm 0.861$ & $12.788 \pm 0.652$ & $6.555 \pm 0.615$ & $3.533 \pm 0.662$ \\
\hline 1994 May 16 & 46.100 & Camb & & & & $8.000 \pm 1.281$ & \\
\hline 1994 May 17 & 47.100 & Camb & & & & $8.400 \pm 1.306$ & \\
\hline 1994 May 19 & 49.252 & VLA-BnA & $3.420 \pm 0.343$ & $15.940 \pm 0.798$ & $11.040 \pm 0.554$ & $5.610 \pm 0.523$ & $2.810 \pm 0.539$ \\
\hline 1994 May 23 & 53.208 & VLA-BnA & & $15.860 \pm 0.809$ & $11.305 \pm 0.570$ & $5.380 \pm 0.547$ & $2.680 \pm 0.722$ \\
\hline 1994 May 24 & 54.060 & Camb & & & & $5.500 \pm 1.141$ & \\
\hline 1994 May 27 & 57.060 & Camb & & & & $5.500 \pm 0.890$ & \\
\hline 1994 May 28 & 58.136 & VLA-BnA & $4.660 \pm 0.466$ & $14.030 \pm 0.704$ & $8.400 \pm 0.425$ & $4.240 \pm 0.473$ & $2.820 \pm 0.645$ \\
\hline 1994 Jun 1 & 62.188 & VLA-BnA & $5.080 \pm 0.508$ & $12.400 \pm 0.626$ & $7.670 \pm 0.392$ & $3.390 \pm 0.441$ & $<2.250^{\mathrm{a}}$ \\
\hline 1994 Jun 17 & 78.165 & VLA-B & $7.190 \pm 0.729$ & $9.640 \pm 0.497$ & $5.860 \pm 0.310$ & $2.920 \pm 0.404$ & $1.910 \pm 0.356$ \\
\hline 1994 Jun 29 & 90.173 & VLA-B & $10.940 \pm 1.103$ & $7.620 \pm 0.388$ & $4.240 \pm 0.221$ & $2.420 \pm 0.359$ & $2.050 \pm 0.596$ \\
\hline 1994 Jul 7 & 98.027 & VLA-B & $10.530 \pm 1.065$ & $7.340 \pm 0.369$ & $4.000 \pm 0.207$ & $2.150 \pm 0.323$ & $<1.620^{\mathrm{a}}$ \\
\hline 1994 Aug 8 & 130.937 & VLA-B & $9.410 \pm 0.951$ & $5.020 \pm 0.262$ & $2.750 \pm 0.150$ & $1.480 \pm 0.348$ & $<2.400^{\mathrm{a}}$ \\
\hline 1994 Aug 22 & 144.959 & VLA-B & $11.480 \pm 1.155$ & $3.760 \pm 0.234$ & $1.930 \pm 0.154$ & $1.620 \pm 0.476$ & $<1.980^{\mathrm{a}}$ \\
\hline 1994 Sep 1 & 154.753 & VLA-B & $9.250 \pm 1.277$ & $2.073 \pm 0.174$ & $1.415 \pm 0.139$ & $<1.320^{\mathrm{a}}$ & $<4.050^{\mathrm{a}}$ \\
\hline 1994 Sep 30 & 183.774 & VLA-CnB & $10.510 \pm 1.196$ & $2.824 \pm 0.213$ & $1.530 \pm 0.097$ & $<1.830^{\mathrm{a}}$ & $<2.160^{\mathrm{a}}$ \\
\hline
\end{tabular}


Table 1

(Continued)

\begin{tabular}{|c|c|c|c|c|c|c|c|}
\hline $\begin{array}{l}\text { Obs. } \\
\text { Date }\end{array}$ & $\begin{array}{l}\text { Age } \\
\text { (days) }\end{array}$ & $\begin{array}{l}\text { Tel. } \\
\text { Config. }\end{array}$ & $\begin{array}{c}S(20 \mathrm{~cm}) \pm \mathrm{rms} \\
(\mathrm{mJy})\end{array}$ & $\begin{array}{c}S(6 \mathrm{~cm}) \pm \mathrm{rms} \\
(\mathrm{mJy})\end{array}$ & $\begin{array}{c}S(3.6 \mathrm{~cm}) \pm \mathrm{rms} \\
(\mathrm{mJy})\end{array}$ & $\underset{(\mathrm{mJy})}{S(2 \mathrm{~cm}) \pm \mathrm{rms}}$ & $\begin{array}{c}S(1.3 \mathrm{~cm}) \pm \mathrm{rms} \\
(\mathrm{mJy})\end{array}$ \\
\hline 1994 Oct 13 & 196.687 & VLA-C & $<2.841^{\mathrm{a}}$ & $2.203 \pm 0.264$ & $1.117 \pm 0.169$ & $<1.140^{\mathrm{a}}$ & $<1.917^{\mathrm{a}}$ \\
\hline 1994 Nov 7 & 221.513 & VLA-C & & $1.257 \pm 0.191$ & $1.220 \pm 0.117$ & $<0.990^{\mathrm{a}}$ & $<2.970^{\mathrm{a}}$ \\
\hline 1994 Dec 5 & 249.708 & VLA-D & & $2.209 \pm 0.357$ & $1.024 \pm 0.176$ & $<2.220^{\mathrm{a}}$ & \\
\hline 1995 Jan 5 & 280.507 & VLA-D & & & & $<1.650^{\mathrm{a}}$ & \\
\hline 1995 Apr 6 & 371.173 & VLA-D & & & & $<1.032^{\mathrm{a}}$ & $<1.920^{\mathrm{a}}$ \\
\hline 1995 Jun 16 & 442.147 & VLA-DnA & $2.397 \pm 0.253$ & $0.855 \pm 0.102$ & & & \\
\hline 1995 Oct 6 & 554.790 & VLA-BnA & $1.087 \pm 0.121$ & $0.503 \pm 0.113$ & $0.391 \pm 0.072$ & $<0.636^{\mathrm{a}}$ & \\
\hline 1996 Jan 12 & 652.500 & VLA-CnB & & $0.460 \pm 0.046$ & & $<0.480^{\mathrm{a}}$ & \\
\hline 1996 Oct 5 & 919.847 & VLA-DnA & $0.580 \pm 0.152$ & $0.250 \pm 0.071$ & $<0.390^{\mathrm{a}}$ & & \\
\hline 1999 Jun 13 & 1900.000 & VLA-DnA & $<0.222^{\mathrm{a}}$ & & & & \\
\hline 2002 Apr 5 & 2927.000 & VLA-A & $0.150 \pm 0.026$ & & & & \\
\hline
\end{tabular}

Note. ${ }^{a}$ All upper limits are $3 \sigma$.

Table 2

Millimeter Flux Density Measurements for SN 1994I

\begin{tabular}{lccccc}
\hline \hline $\begin{array}{l}\text { Obs. } \\
\text { Date }\end{array}$ & $\begin{array}{c}\text { Age } \\
(\text { days })\end{array}$ & Tel. & $\begin{array}{c}\text { Flux Density }(S) \pm \mathrm{rms}^{\mathrm{a}} \\
(\mathrm{mJy})\end{array}$ & $\begin{array}{c}\text { Frequency } \\
(\mathrm{GHz})\end{array}$ & Reference \\
\hline 1994 Mar 31 & $=0.00$ & & & & \\
1994 Apr 5 & 5.45 & OVRO & $\begin{array}{c}11.0 \pm 0.6 \\
<12^{\mathrm{b}}\end{array}$ & 99 & Phillips et al. (1994) \\
1994 Apr 6 & 6.45 & OVRO & 218 & Phillips et al. (1994) \\
\hline
\end{tabular}

Notes.

${ }^{a}$ Quoted errors are taken from the original references.

${ }^{\mathrm{b}}$ All upper limits are $3 \sigma$.

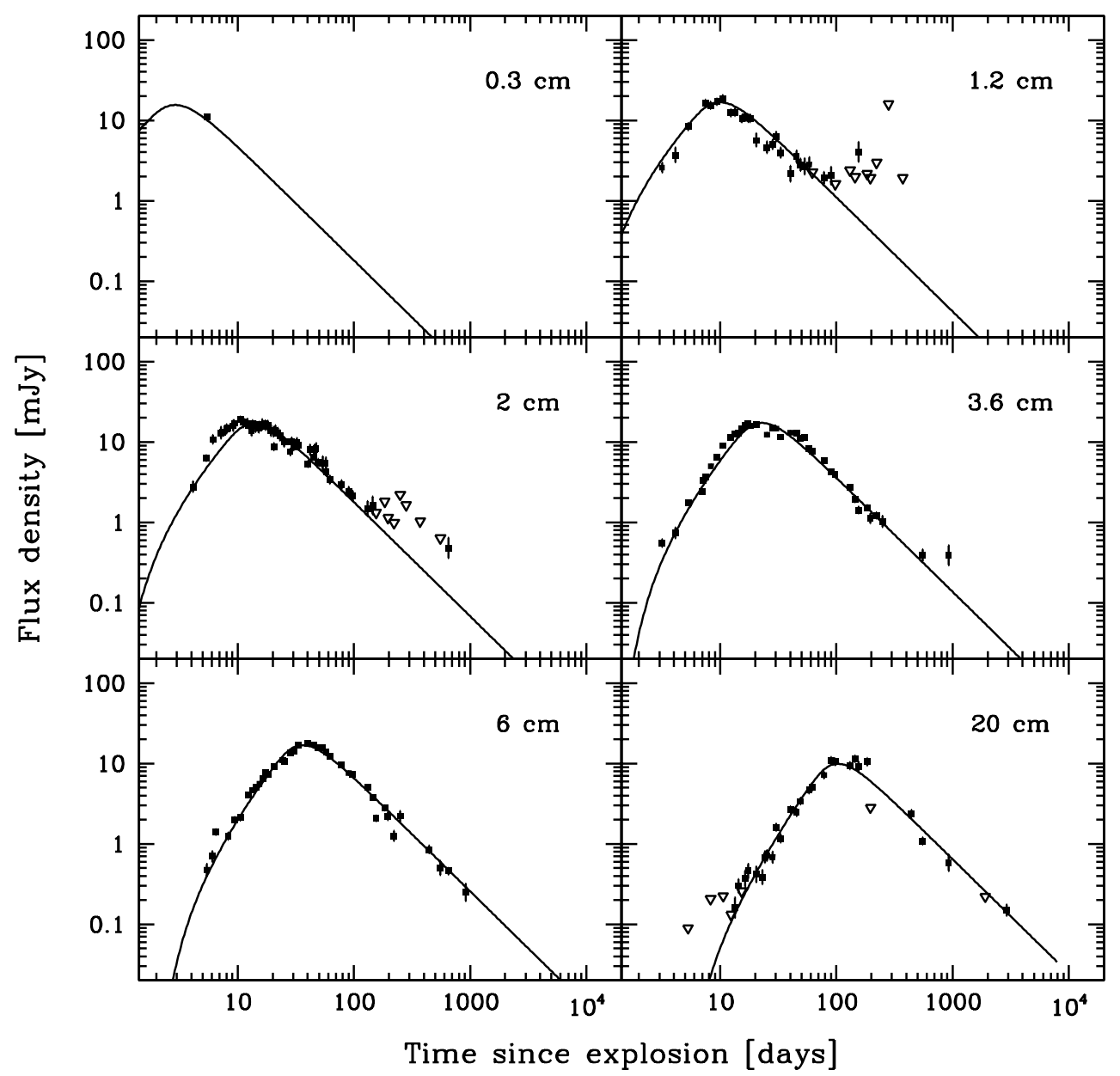

Figure 1. Radio light curves for SN 1994I for top left $0.3 \mathrm{~cm}$, top right $1.2 \mathrm{~cm}$, middle left $2 \mathrm{~cm}$, middle right $3.6 \mathrm{~cm}$, lower left $6 \mathrm{~cm}$, and lower right $20 \mathrm{~cm}$. The lines on all figures represent the best-fit model as described in the text with the parameters listed in Table 4. 
Table 3

VLA Calibrator Flux Density Measurements ${ }^{\mathrm{a}}$

\begin{tabular}{|c|c|c|c|c|c|}
\hline $\begin{array}{l}\text { Obs. } \\
\text { Date }\end{array}$ & $\begin{array}{c}S(20 \mathrm{~cm}) \\
(\mathrm{mJy})\end{array}$ & $\begin{array}{c}S(6 \mathrm{~cm}) \\
(\mathrm{mJy})\end{array}$ & $\begin{array}{c}S(3.6 \mathrm{~cm}) \\
(\mathrm{mJy})\end{array}$ & $\begin{array}{c}S(2 \mathrm{~cm}) \\
(\mathrm{mJy})\end{array}$ & $\begin{array}{c}S(1.3 \mathrm{~cm}) \\
\quad(\mathrm{mJy})\end{array}$ \\
\hline 1994 Apr 3 & & & $0.539^{b}$ & & $0.338^{\mathrm{b}}$ \\
\hline 1994 Apr 4 & & & $1.014^{\mathrm{c}}$ & $0.953^{c}$ & $0.830^{\mathrm{c}}$ \\
\hline 1994 Apr 5 & 0.904 & 1.038 & 1.014 & 0.953 & 0.830 \\
\hline 1994 Apr 6 & & $1.020^{\mathrm{c}}$ & & & \\
\hline 1994 Apr 7 & & & 0.994 & $0.959^{c}$ & $0.880^{\mathrm{c}}$ \\
\hline 1994 Apr 8 & $0.899^{\mathrm{c}}$ & $1.025^{\mathrm{c}}$ & $1.000^{\mathrm{c}}$ & $0.954^{\mathrm{c}}$ & $0.875^{\mathrm{c}}$ \\
\hline 1994 Apr 9 & & $1.040^{\mathrm{c}}$ & $1.019^{c}$ & $0.980^{\mathrm{c}}$ & $0.910^{\mathrm{c}}$ \\
\hline 1994 Apr 10 & 0.894 & 1.013 & 0.987 & 0.956 & 0.920 \\
\hline 1994 Apr 12 & $0.887^{\mathrm{c}}$ & $1.006^{\mathrm{c}}$ & $0.974^{\mathrm{c}}$ & $0.940^{c}$ & $0.864^{\mathrm{c}}$ \\
\hline 1994 Apr 13 & 0.884 & 1.017 & 0.993 & 0.955 & 0.865 \\
\hline 1994 Apr 14 & $0.875^{\mathrm{c}}$ & 1.026 & $1.027^{\mathrm{c}}$ & $0.976^{\mathrm{c}}$ & \\
\hline 1994 Apr 15 & $0.875^{\mathrm{c}}$ & $1.017^{\mathrm{c}}$ & $1.029^{c}$ & $0.993^{c}$ & $0.839^{c}$ \\
\hline 1994 Apr 16 & $0.875^{\mathrm{c}}$ & $1.017^{\mathrm{c}}$ & $1.029^{c}$ & $0.993^{c}$ & $0.839^{c}$ \\
\hline 1994 Apr 17 & $0.875^{\mathrm{c}}$ & $1.060^{\mathrm{c}}$ & $1.032^{\mathrm{c}}$ & $0.976^{\mathrm{c}}$ & $0.810^{\mathrm{c}}$ \\
\hline 1994 Apr 18 & & 0.984 & 0.963 & 0.930 & 0.901 \\
\hline 1994 Apr 20 & $0.875^{\mathrm{c}}$ & $1.005^{\mathrm{c}}$ & $0.964^{\mathrm{c}}$ & $0.918^{c}$ & 0.773 \\
\hline 1994 Apr 23 & $0.875^{\mathrm{c}}$ & & & & \\
\hline 1994 Apr 24 & $0.875^{\mathrm{c}}$ & $1.015^{\mathrm{c}}$ & & & \\
\hline 1994 Apr 25 & 0.904 & 1.015 & 0.946 & & 0.689 \\
\hline 1994 Apr 28 & 0.866 & 0.995 & 0.937 & 0.881 & 0.683 \\
\hline 1994 Apr 30 & $0.887^{\mathrm{c}}$ & $0.993^{\mathrm{c}}$ & $0.958^{\mathrm{c}}$ & $0.890^{\mathrm{c}}$ & $0.792^{\mathrm{c}}$ \\
\hline 1994 May 3 & 0.905 & 0.992 & 0.940 & & 0.900 \\
\hline 1994 May 10 & 0.890 & 0.946 & 0.930 & 0.859 & 0.846 \\
\hline 1994 May 15 & 0.899 & 0.983 & 0.949 & 0.900 & 0.816 \\
\hline 1994 May 19 & 0.868 & 0.948 & 0.920 & 0.830 & 0.764 \\
\hline 1994 May 23 & & 0.986 & 0.933 & 0.863 & 0.797 \\
\hline 1994 May 28 & 0.872 & 0.976 & 0.939 & 0.878 & 0.822 \\
\hline 1994 Jun 1 & 0.894 & 0.996 & 0.963 & 0.874 & 0.905 \\
\hline 1994 Jun 17 & 0.894 & 0.991 & 0.957 & 0.875 & 0.793 \\
\hline 1994 Jun 29 & 0.883 & 0.958 & 0.918 & 0.829 & 0.786 \\
\hline 1994 Jul 7 & 0.882 & 0.927 & 0.885 & 0.783 & 0.753 \\
\hline 1994 Aug 8 & 0.881 & 0.912 & 0.879 & 0.846 & 0.827 \\
\hline 1994 Aug 22 & 0.926 & 0.863 & 0.784 & 0.635 & 0.633 \\
\hline 1994 Sep 1 & $0.906^{\mathrm{c}}$ & $0.852^{\mathrm{c}}$ & $0.807^{\mathrm{c}}$ & 0.657 & 0.665 \\
\hline 1994 Sep 30 & 0.885 & 0.831 & 0.830 & 0.654 & 0.528 \\
\hline 1994 Oct 13 & $0.840^{\mathrm{c}}$ & $0.834^{\mathrm{c}}$ & $0.822^{\mathrm{c}}$ & $0.705^{\mathrm{c}}$ & $0.610^{\mathrm{c}}$ \\
\hline 1994 Nov 7 & & 0.826 & 0.812 & 0.755 & 0.693 \\
\hline 1994 Dec 5 & & 0.832 & 0.782 & 0.680 & \\
\hline 1995 Jan 5 & & & & 0.726 & \\
\hline 1995 Apr 6 & & & & 0.697 & 0.634 \\
\hline 1995 Jun 16 & 0.760 & 0.764 & & & \\
\hline 1995 Oct 6 & 0.705 & 0.750 & 0.738 & 0.702 & \\
\hline 1996 Jan 12 & & 0.726 & & 0.697 & \\
\hline 1996 Oct 5 & 0.622 & 0.775 & 0.792 & & \\
\hline 1999 Jun 13 & 0.498 & & & & \\
\hline 2002 Apr 5 & $0.537^{\mathrm{d}}$ & & & & \\
\hline
\end{tabular}

Notes.

${ }^{a}$ Unless otherwise noted, the flux density measurements are for $\mathrm{J} 1418+546$.

${ }^{\mathrm{b}}$ Flux density measurements are for J1415+463.

${ }^{c}$ Interpolated from nearby measurements.

${ }^{\mathrm{d}}$ Flux density measurement is for $1327+434$.

for four pairs of bands $(1.2-2 \mathrm{~cm}, 2-3.6 \mathrm{~cm}, 3.6-6 \mathrm{~cm}$, and $6-20 \mathrm{~cm})$. The parameters listed in Table 4 from the best-fit light curves allow calculation of the model spectral index evolution curves and these are also shown in Figure 2. At the earliest times, the observed spectral indices exceed the maximum value spectral index $(\alpha=2.5)$ possible for a purely synchrotron selfabsorption (SSA) source, suggesting that at least some thermal absorption is present in SN 1994I.

The overall model fits to the spectral index evolution are quite good, with the only exception being the model $1.2-2 \mathrm{~cm}$
Table 4

Best-fit Parameters for SN 1994I and Comparison with SN 1993J

\begin{tabular}{|c|c|c|}
\hline Parameter & SN 1994I & SN $1993 J^{a}$ \\
\hline$\overline{K_{1}}$ & $4.78 \times 10^{3}$ & $4.8 \times 10^{3}$ \\
\hline$\alpha$ & -1.22 & -0.81 \\
\hline$\beta$ & -1.42 & -0.73 \\
\hline$K_{2}$ & $3.0 \times 10^{1}$ & $1.6 \times 10^{2}$ \\
\hline$\delta$ & $\equiv-3.00^{\mathrm{b}}$ & -1.88 \\
\hline$K_{3}$ & $\equiv 0^{\mathrm{b}}$ & $4.3 \times 10^{5}$ \\
\hline$\delta^{\prime}$ & $\ldots$ & -2.83 \\
\hline$K_{4}$ & $4.55 \times 10^{-2}$ & $\equiv 0$ \\
\hline$K_{5}$ & $6.90 \times 10^{5}$ & $2.62 \times 10^{3}$ \\
\hline$\delta^{\prime \prime}$ & -4.08 & -2.05 \\
\hline$K_{6}$ & $9.96 \times 10^{3}$ & $\equiv 0$ \\
\hline$\delta^{\prime \prime \prime}$ & -2.63 & $\cdots$ \\
\hline Distance $(\mathrm{Mpc})$ & 8.9 & 3.63 \\
\hline Date of explosion $\left(t_{0}\right)$ & 1994 Mar 31 & 1993 Mar 28 \\
\hline Time to $\mathrm{S}_{6 \mathrm{~cm} \text { peak }}$ (days) & 34.4 & 133 \\
\hline$S_{6 \mathrm{~cm} \text { peak }}(\mathrm{mJy})$ & 16.2 & 96.9 \\
\hline$L_{6 \mathrm{~cm} \text { peak }}\left(\mathrm{erg} \mathrm{s}^{-1} \mathrm{~Hz}^{-1}\right)$ & $1.5 \times 10^{27}$ & $1.5 \times 10^{27}$ \\
\hline$\dot{M}\left(M_{\odot} \mathrm{yr}^{-1}\right)$ & $>1.4 \times 10^{-4 \mathrm{c}}$ & $0.5-5.9 \times 10^{-6 \mathrm{~d}}$ \\
\hline
\end{tabular}

Notes.

a Weiler et al. (2007).

b Adopted. Because the amount of thermal material surrounding SN 1994I is so low, it is not possible to determine $\delta$ and $K_{3}$ from the data fitting, and nominal values were adopted.

${ }^{c}$ Under the assumption of a Wolf-Rayet progenitor; see Section 4.6.

${ }^{\mathrm{d}}$ Weiler et al. (2007) found that the mass-loss rate from SN 1993J increased from $\sim 5.4 \times 10^{-7} M_{\odot} \mathrm{yr}^{-1}$ at the time of shock breakout to $\sim 5.9 \times 10^{-6} M_{\odot} \mathrm{yr}^{-1}$ around day $\sim 3100$ ( $\sim 8000$ yr before explosion).



Figure 2. Spectral index evolution for SN 1994I for top left $1.2-2 \mathrm{~cm}$, top right $2-3.6 \mathrm{~cm}$, lower left $3.6-6 \mathrm{~cm}$, and lower right $6-20 \mathrm{~cm}$. The lines on all figures represent the best-fit model as described in the text with the parameters listed in Table 4.

spectral index at early times, which is systematically higher than the observed values. This deviation, which was found also for the case of SN 1993J (Weiler et al. 2007), suggests that, at the highest frequencies, the intrinsic radio spectrum has a somewhat 

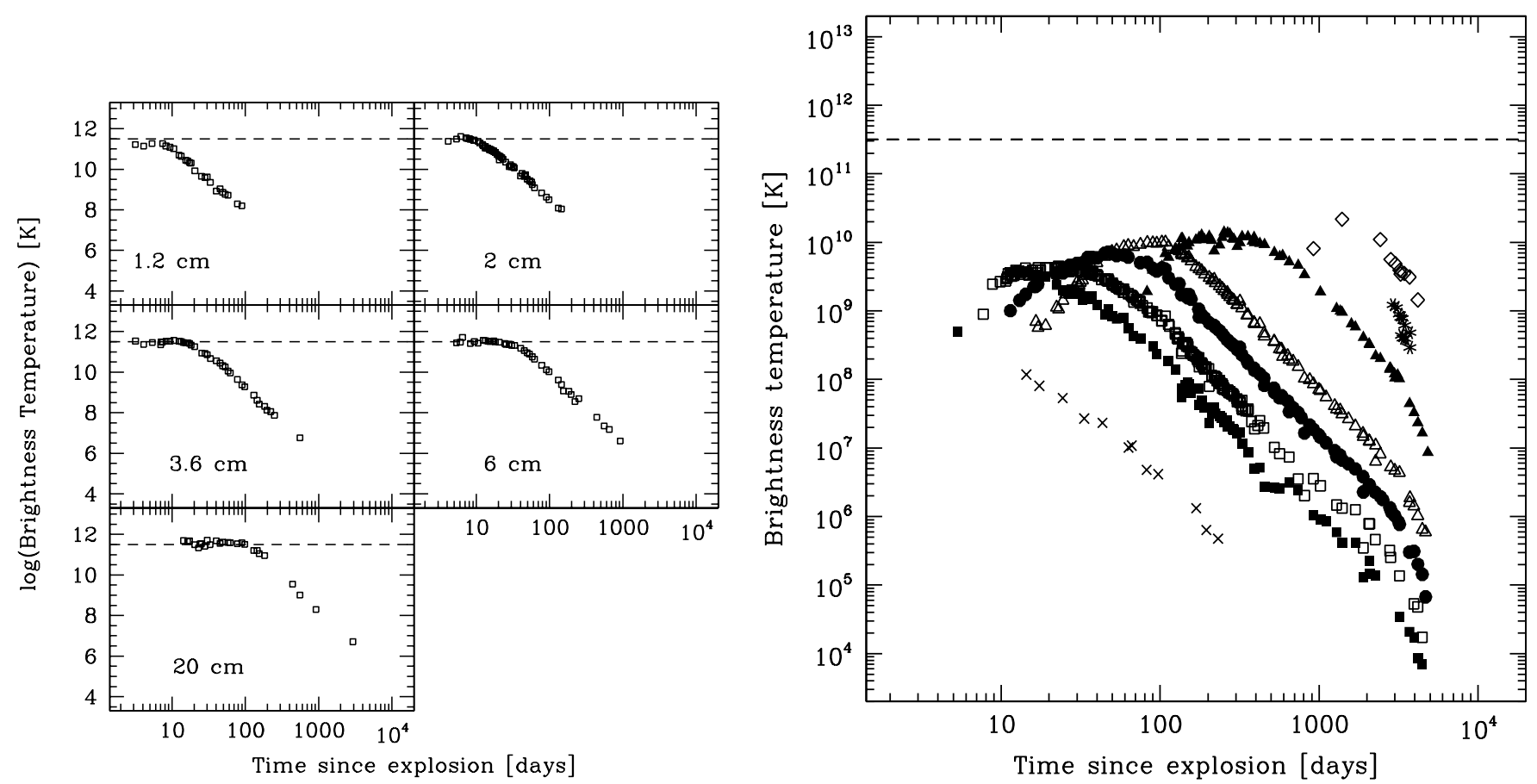

Figure 3. Left: the brightness temperature $\left(T_{B}\right)$ evolution, uncorrected for thermal absorption, for SN 1994I for top left $1.2 \mathrm{~cm}$, top right $2 \mathrm{~cm}$, middle left $3.6 \mathrm{~cm}$, middle right $6 \mathrm{~cm}$, and lower left $20 \mathrm{~cm}$; right: the brightness temperature $\left(T_{B}\right)$ evolution, uncorrected for thermal absorption, for SN 1993J (Weiler et al. 2007) for, from left to right, $0.3 \mathrm{~cm}$ (cross), $1.2 \mathrm{~cm}$ (filled square), $2 \mathrm{~cm}$ (open square), $3.6 \mathrm{~cm}$ (filled circle), $6 \mathrm{~cm}$ (open triangle), $20 \mathrm{~cm}$ (filled triangle), $49 \mathrm{~cm}$ (star), and $90 \mathrm{~cm}$ (open diamond). The horizontal dashed lines in all plots denote the limiting value of $T_{B} \simeq 3 \times 10^{11} \mathrm{~K}$ (Kellermann \& Pauliny-Toth 1969; Readhead 1994).

flatter spectral index than the best-fit value determined by the longer wavelength data.

\subsection{Brightness Temperature}

This extensive data set can also be used to estimate the intrinsic brightness temperature of the SN 1994I radio emission which can be derived from the ratio of the observed radio flux density to the solid angle $\Omega$ of the emitting region $\left(T_{B} \propto\right.$ $\left.S_{\text {obs }} / \Omega\right)$.

Since SN 1994I was not radio bright enough, it was not possible to directly measure its angular size by means of very long baseline interferometry observations, as had been possible for SN 1993J (see, e.g., Marcaide et al. 2009, and references therein), but the physical size of the SN shock can be estimated from the optically measured expansion velocity and distance to the SN. For SN 1994I, published expansion velocities at early times range between $\sim 16,500 \mathrm{~km} \mathrm{~s}^{-1}$ (Filippenko et al. 1995; Millard et al. 1999) from the absorption troughs of He I $\lambda 10830$, through $\sim 20,000 \mathrm{~km} \mathrm{~s}^{-1}$ (Millard et al. 1999) as seen for Si II, up to almost $30,000 \mathrm{~km} \mathrm{~s}^{-1}$, that is the extent measured by Filippenko et al. (1995) for the blue wing of the He I $\lambda 10830$ absorption line. We shall adopt a compromise expansion velocity of $20,000 \mathrm{~km} \mathrm{~s}^{-1}$ and, following Immler et al. (2002), we shall assume that it is constant with time. Therefore, the radius $r$ of the emitting region can be estimated as

$$
r=1.73 \times 10^{14}(t / 1 \text { day }) \mathrm{cm}
$$

which, for an adopted distance $D$ to SN 1994I of $8.9 \mathrm{Mpc}$, gives an angular size of

$$
\theta=1.29(t / 1 \text { day }) \mu \text { as }
$$

and an estimated brightness temperature of

$$
T_{B}=3.5 \times 10^{11} S_{\text {obs }}(\mathrm{mJy}) \lambda^{2}(\mathrm{t} / 1 \text { day })^{-2} \mathrm{~K} .
$$

The resulting brightness temperature evolution is shown in Figure 3 (left). The corresponding brightness temperature evolution curves for SN 1993J from Weiler et al. (2007) are also shown in Figure 3 (right).

From these brightness temperature estimates, we can constrain the value of external absorption due to a presupernova stellar wind by requiring that $T_{B}$ not exceed the limit of $\sim 3 \times 10^{11} \mathrm{~K}$ (Kellermann \& Pauliny-Toth 1969; Readhead 1994) where the radio emission is quenched by inverse-Compton scattering. This implies that any value of $K_{2}$ (see Section 3.1) higher than $\sim 30$ would yield $T_{B}$ curves in strong disagreement with such a physical requirement and puts an upper limit on the amount of external thermal absorption by a presupernova mass loss in a dense stellar wind.

\section{RADIO SUPERNOVA MODELS}

\subsection{Parameterized Model}

Weiler et al. (1986) discuss the common properties of RSNe, including non-thermal synchrotron emission with high brightness temperature, turn-on delay at longer wavelengths, powerlaw decline with index $\beta$ after maximum, and spectral index $\alpha\left(\mathrm{S} \propto v^{+\alpha}\right)$ asymptotically decreasing to an optically thin value. Weiler et al. $(1986,1990)$ have shown that the "minishell" model of Chevalier (1982a, 1982b), with modifications by Weiler et al. (1990), adequately describes previously known RSNe. In this model, the relativistic electrons and enhanced magnetic fields necessary for synchrotron emission are generated by the SN shock interacting with a relatively high density circumstellar envelope which has been ionized and heated by the initial UV/X-ray flash. Figure 4 (taken from Weiler et al. 2002) shows a, not to scale, greatly simplified cartoon of the vicinity of a supernova explosion. This dense cocoon is presumed to have been established by a constant mass-loss $(\dot{M})$ rate, 


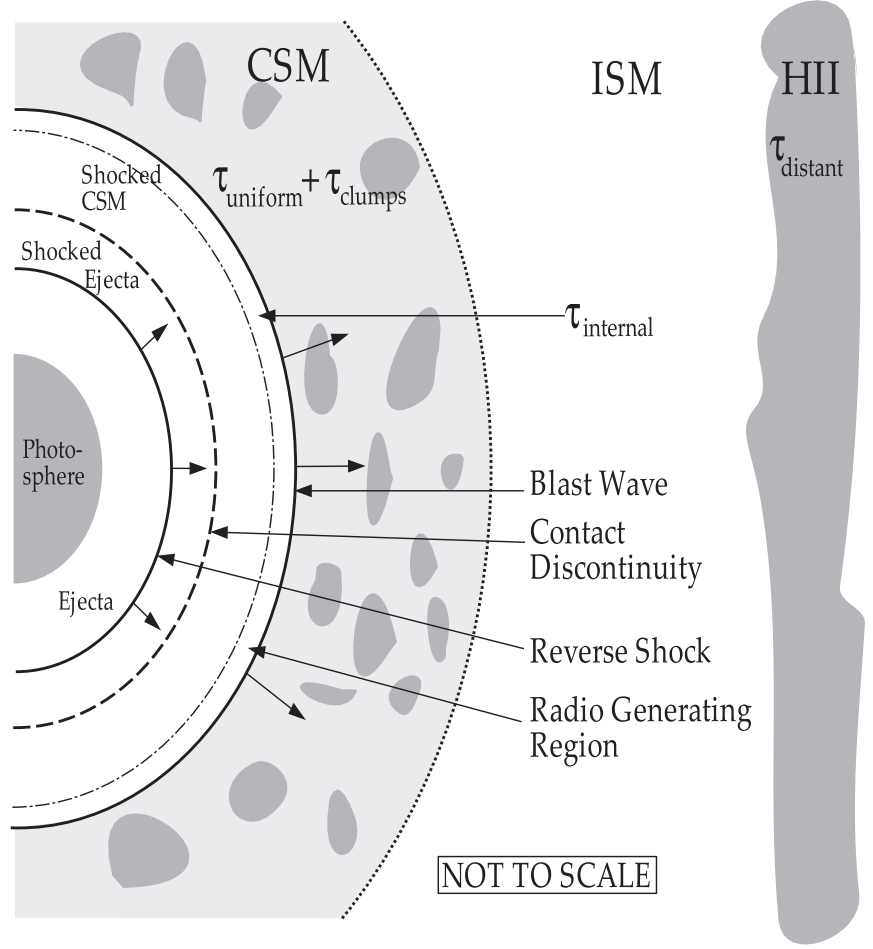

Figure 4. Cartoon (taken from Weiler et al. (2002)), not to scale, of the supernova and its shocks, along with the stellar wind established CSM, the interstellar medium (ISM), and more distant ionized hydrogen (H II) absorbing gas. The radio emission is thought to arise near the blast wave front. The expected locations of the several absorbing terms in Equations (5)-(12) are illustrated.

constant velocity $(w)$ wind (i.e., $\rho_{\mathrm{CSM}} \propto r^{-2}$ ) from a massive $\left(>8 M_{\odot}\right)$ stellar progenitor or companion. This ionized CSM can also be the source of initial thermal absorption (free-free absorption, FFA) although Chevalier (1998) has suggested that non-thermal, SSA may be a significant source of absorption in the early phases of some RSNe. For the case of FFA, the rapid rise in radio flux density results from the shock overtaking progressively more of the wind matter, leaving less of it along the line of sight to the observer to absorb the more slowly decreasing synchrotron emission from the shock region. In the case of SSA, the non-thermal absorption decreases as the emitting region expands and decreases in density. Montes et al. (1997) also allow for the possibility of intervening $\mathrm{H}$ II along the line of sight to the RSN, providing a constant thermal absorption of the radio emission.

To parameterize the basic characteristics of RSNe, we adopt the most recent RSN discussion of Weiler et al. (2002) and Sramek \& Weiler $(2003)^{8}$ and illustrate the regions of the supernova being parameterized in a cartoon shown in Figure 4 from, e.g., Weiler et al. (2002):

$$
\begin{aligned}
S(\mathrm{mJy})= & K_{1}\left(\frac{v}{5 \mathrm{GHz}}\right)^{\alpha}\left(\frac{t-t_{0}}{1 \text { day }}\right)^{\beta} e^{-\tau_{\text {external }}} \\
& \times\left(\frac{1-e^{-\tau_{\mathrm{CSM}} \text { clumps }}}{\tau_{\mathrm{CSM}_{\text {clumps }}}}\right)\left(\frac{1-e^{-\tau_{\text {internal }}}}{\tau_{\text {internal }}}\right)
\end{aligned}
$$

with

$$
\tau_{\text {external }}=\tau_{\mathrm{CSM}_{\text {homogeneous }}}+\tau_{\text {distant }},
$$

8 Even though this model has been previously described in papers such as the references given above, we list it again for completeness, for the reader's convenience, and for explanation of the parameters listed in Table 4. where

$$
\begin{gathered}
\tau_{\mathrm{CSM}_{\text {homogeneous }}}=K_{2}\left(\frac{v}{5 \mathrm{GHz}}\right)^{-2.1}\left(\frac{t-t_{0}}{1 \mathrm{day}}\right)^{\delta} \\
\tau_{\text {distant }}=K_{4}\left(\frac{v}{5 \mathrm{GHz}}\right)^{-2.1}
\end{gathered}
$$

and

$$
\tau_{\mathrm{CSM}_{\mathrm{clumps}}}=K_{3}\left(\frac{v}{5 \mathrm{GHz}}\right)^{-2.1}\left(\frac{t-t_{0}}{1 \text { day }}\right)^{\delta^{\prime}}
$$

with $K_{1}, K_{2}, K_{3}$, and $K_{4}$ determined from fits to the data and corresponding, formally, to the flux density $\left(K_{1}\right)$, homogeneous $\left(K_{2}, K_{4}\right)$, and clumpy or filamentary $\left(K_{3}\right)$ FFA at $5 \mathrm{GHz}$ one day after the explosion date $t_{0}$. The terms $\tau_{\mathrm{CSM}_{\text {homogeneos }}}$ and $\tau_{\mathrm{CSM}_{\text {clumps }}}$ describe the attenuation due to local, homogeneous FFA CSM and clumpy or filamentary FFA CSM, respectively, that are near enough to the SN progenitor that they are altered by the rapidly expanding SN blast wave. The $\tau_{\mathrm{CSM}_{\text {homogeneous }}}$ FFA is produced by an ionized medium that completely covers the emitting source ("homogeneous external absorption"), and the $\left(1-e^{\left.-\tau_{\mathrm{CSM}_{\mathrm{clumps}}}\right)} \tau_{\mathrm{CSM}_{\text {clumps }}}^{-1}\right.$ term describes the attenuation produced by an inhomogeneous FFA medium ("clumpy absorption;" see Natta \& Panagia 1984, for a more detailed discussion of attenuation in inhomogeneous media). The $\tau_{\text {distant }}$ term describes the attenuation produced by a homogeneous FFA medium which completely covers the source but is so far from the SN progenitor that it is not affected by the expanding SN blast wave and is consequently constant in time. All external and clumpy absorbing media are assumed to be purely thermal, singly ionized gas which absorbs via FFA with frequency dependence $v^{-2.1}$ in the radio. The parameters $\delta$ and $\delta^{\prime}$ describe the time dependence of the optical depths for the local homogeneous and clumpy or filamentary media, respectively.

Since it is physically realistic and is needed in some RSNe where radio observations have been obtained at early times and high frequencies, Equation (5) also includes the possibility for an internal absorption term. ${ }^{9}$ This internal absorption $\left(\tau_{\text {internal }}\right)$ term may consist of two parts-SSA $\left(\tau_{\text {internalssa }_{\text {s }}}\right)$, and thermal FFA $\left(\tau_{\text {internal }_{\text {FFA }}}\right)$ due to ionized gas mixed with non-thermal emission.

$$
\begin{gathered}
\tau_{\text {internal }}=\tau_{\text {internal }_{\mathrm{SSA}}}+\tau_{\text {internal }_{\mathrm{FFA}}} \\
\tau_{\text {internal }_{\mathrm{SSA}}}=K_{5}\left(\frac{v}{5 \mathrm{GHz}}\right)^{\alpha-2.5}\left(\frac{t-t_{0}}{1 \text { day }}\right)^{\delta^{\prime \prime}} \\
\tau_{\text {internal }_{\mathrm{FFA}}}=K_{6}\left(\frac{v}{5 \mathrm{GHz}}\right)^{-2.1}\left(\frac{t-t_{0}}{1 \text { day }}\right)^{\delta^{\prime \prime \prime}}
\end{gathered}
$$

with $K_{5}$ corresponding, formally, to the internal, non-thermal $\left(v^{\alpha-2.5}\right) \mathrm{SSA}$ and $K_{6}$, corresponding, formally, to the internal thermal $\left(v^{-2.1}\right)$ FFA mixed with non-thermal emission, at $5 \mathrm{GHz}$ one day after the explosion date $t_{0}$. The parameters $\delta^{\prime \prime}$ and $\delta^{\prime \prime \prime}$ describe the time dependence of the optical depths for the SSA and FFA internal absorption components, respectively.

\footnotetext{
9 Note that for simplicity an internal absorber attenuation of the form

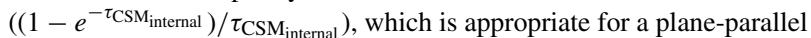
geometry, is used instead of the more complicated expression (e.g., Osterbrock 1974) valid for the spherical case. The assumption does not affect the quality of the analysis because, to within 5\% accuracy, the optical depth obtained with the spherical case formula is simply three-fourths of that obtained with the plane-parallel slab formula.
} 
Application of this basic parameterization has been shown to be effective in describing the physical characteristics of the presupernova system, its CSM, and its final stages of evolution before explosion for objects ranging from the two decades of monitoring the complex radio emission from SN 1979C (Montes et al. 2000), through the unusual SN 1998bw (GRB 980425; Weiler et al. 2001a), to most recent $\gamma$-ray bursters/Type Ib/c SNe (Weiler et al. 2002, 2003).

\section{DISCUSSION}

\subsection{Application of Models to SN 1994I}

As was clearly demonstrated by Weiler et al (2007) for the case of SN 1993J, in order to obtain a meaningful representation of radio observations of a supernova, a model must be able to account simultaneously for three different aspects, namely, (1) the light curves measured at several epochs and in several distinct radio bands, (2) the corresponding spectral indices computed for pairs of bands at adjacent frequencies, and (3) the brightness temperatures, which at all times should be lower than the well-established limit of $3 \times 10^{11} \mathrm{~K}$ (Kellermann \& Pauliny-Toth 1969; Readhead 1994).

\subsection{SN 1994I Radio Light Curves}

We have excellent time coverage of the radio emission from SN 1994I at 5 frequencies, from $22 \mathrm{GHz}$ down to $1.4 \mathrm{GHz}$. That enables us to make a detailed study of its radio properties. In particular, the rising branch of the light curve at early times, where absorption $(\tau \gg 1)$ is still important, is well defined in all five main bands. Also, the flux density evolution is well characterized by a power-law decay at late times, in the optically thin phases. Therefore, we are in an excellent position to identify the main processes operating in this source and determine their parameters. Actually, the best-fit curves as described by the parameters in Table 4 and shown in Figure 1 agree quite well with the observations, with an overall $\chi^{2}=4.42$ per degree of freedom.

From this rather good parameterization of the radio emission from SN 1994I, we conclude that the radio light curves are not well described by a purely thermal absorption model such as that given by Equations (5)-(9). In particular, the requirement for non-zero $K_{5}$ and $K_{6}$ parameter values implies the existence of significant SSA. In fact, the early optical depth is dominated almost entirely by the SSA $\left(K_{5}\right)$ component, and the FFA component $\left(K_{6}\right)$ makes a significant contribution only as the emission becomes more optically thin and the brightness temperature begins to drop. As predicted by Chevalier (1998), there does not appear to be a highly-structured, thick CSM present since there is no need for a large $K_{2}$ or non-zero $K_{3}$ term. The necessity of a $K_{4}$ term (i.e., thermal absorption by foreground $\mathrm{H}$ II gas; see Section 4.5) is consistent with recent deep $20 \mathrm{~cm}$ observations that indicate diffuse radio emission from the host galaxy in the region near the supernova (Maddox et al. 2007). Further evidence for substantial amounts of $\mathrm{H}$ II gas is provided by the $\mathrm{H} \alpha$ emission detected on HST images of the immediate vicinity of SN 1994I (Maddox 2006; Maddox et al. 2007).

It is interesting to note that SN 1994I shows a dip around day 27 in its flux density at centimeter wavelengths (frequencies from $4.9 \mathrm{GHz}$ to $22 \mathrm{GHz}$ ). While less pronounced than that noted for the unusual Type Ib/c SN 1998bw, which is related to the $\gamma$-ray burster GRB 980425, it occurs on a similar timescale (see Kulkarni et al. 1998, and http://www.narrabri.atnf.csiro.au/public/grb/grb980425/). This early "dip" followed by "re-brightening" for SN 1998bw has variously been explained as due to a slowing and re-energization of the SN blast wave (Li \& Chevalier 1999) or, more likely, as structure in the circumstellar absorbing material (Weiler et al. 2001a, 2001b).

Considering the light curves shown in Figure 1 in more detail, even though the fitted model provides a good representation the agreement is never quite perfect, mostly because the model is rather simplified and also because there may be systematic deviations from our basic assumptions. In order to analyze the residuals between the model and the observations, we express them as logarithms of the ratios of observed to model fluxes and plot them in Figure 5 (left). A similar analysis helped us in other RSNe to reveal unsuspected features such as a periodic flux modulation for SN 1979C (Weiler et al. 1992; Schwarz \& Pringle 1996) possibly due to the presence of a companion, as well as evidence for interacting winds in SN 2001ig (Ryder et al. 2004).

The residuals for SN 1994I are displayed separately for individual bands in Figure 5 (left) and merged in a single plot in Figure 5 (right). As expected, they show scatter inherent to nonnegligible measurement errors and to a simplified description of a complicated phenomenon. However, a systematic feature is apparent, essentially with the same timing and comparable amplitudes, at all bands. In particular, in Figure 5 (right) we can see a somewhat broad trough, with an apparent local minimum, which is about 0.2 deep in the logarithm and occurring $\sim 27$ days after explosion. This trough extends from $\sim 10$ up to $\sim 55$ days after explosion. Since this local minimum is present at all frequencies with similar amplitude, it must represent a genuine decrease in the intrinsic emission rather than being the result of additional, temporary absorption along the line of sight. In a spherically symmetric model it would correspond to a temporary decrease of the average density of the CSM by $\sim 0.1$ in the logarithm of the ratio, or a factor of $\sim 0.8$ decrease in the CSM density, with a duration of $\sim 45$ days.

Although a temporary decrease in the mass-loss rate from the presupernova star cannot be ruled out, we feel it worthwhile to also consider a model in which the SN 1994I progenitor was a Wolf-Rayet (W-R) star in a wide binary system whose companion was also an early-type star (most likely a B-type star on the main sequence). Such a binary system could create non-spherical CSM density structures by the interacting winds of the system stars (see, e.g., Schwarz \& Pringle 1996). If such were the case, for SN 1994I the observed minimum in the residuals would correspond to a separation between the two stars of $\sim 4.7 \times 10^{15} \mathrm{~cm}$ or $\sim 310 \mathrm{AU}$ (see also SN 1979C (Weiler et al. 1992; Schwarz \& Pringle 1996) where the radio emission modulation is possibly due to the presence of a companion, as well as evidence for interacting winds in SN 2001ig (Ryder et al. 2004)).

\subsection{SN 1994I Spectral Index Evolution}

Examination of the spectral index evolution in Figure 2 shows three things: (1) the model listed in Table 4 generally describes the spectral index evolution quite well except at $1.2-2 \mathrm{~cm}$. Apparently, at higher frequencies the model is beginning to fail. (2) The spectral index evolution, particularly at the first part at $3.6-6 \mathrm{~cm}$, requires some (small) amount of thermal absorption since SSA can never generate a spectral index exceeding +2.5. (3) There is no evidence for systematic spectral index change of the emission within the available observations. While 

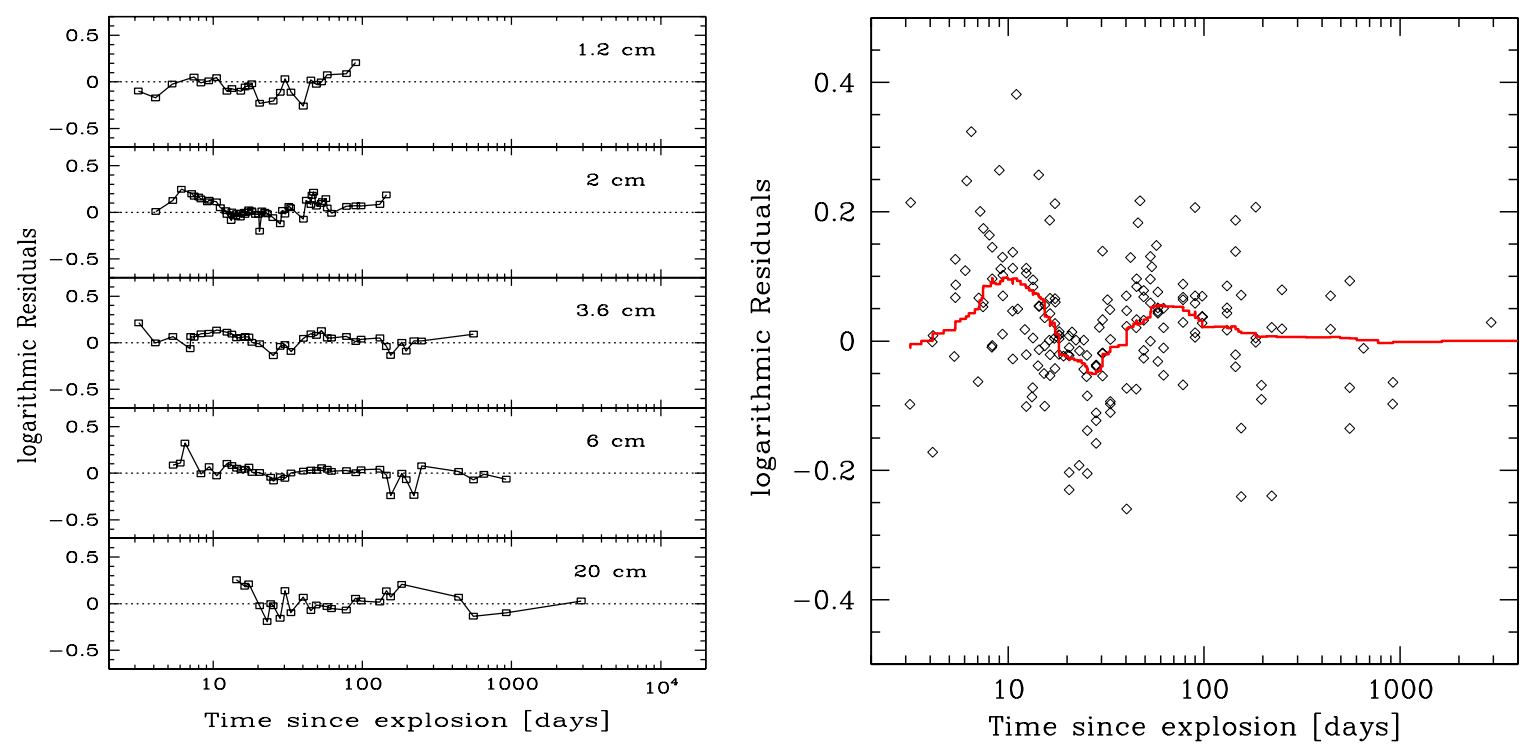

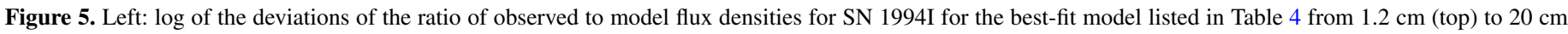
(bottom); right: composite of the deviations at all wavelengths shown on the left, with a smoothed curve added to guide the eye.

(A color version of this figure is available in the online journal.)

short-term fluctuations of the spectral index cannot be ruled out, none appear to occur at the same epoch at all frequencies.

\subsection{Brightness Temperature}

Figure 3 shows the estimated brightness temperatures for each frequency versus time for SNe 1994I (left) and 1993J (right; from Weiler et al. 2007). While the precise temperature measurements depend on the distance used and the assumed (or, for SN 1993J, measured) shock wave speed, it is clear that the two plots have a systematic difference at early times. In the case of SN 1994I, the temperatures for each frequency appear to remain approximately constant near the maximum possible value of $3 \times 10^{11} \mathrm{~K}$ (see Section 2.3) until the source becomes optically thin. However, the measured brightness temperatures for SN 1993J at the earliest times increase for each frequency until the emission becomes optically thin. This is evidence that the dominant absorption mechanism is different between these two SNe, with the primary absorption mechanism for SN 1993J not directly related to the emission mechanism (i.e., external thermal electrons), while the primary absorption mechanism for SN 1994I is physically associated with the emission mechanism (i.e., synchrotron electrons). This simple comparison makes it clear that SSA is dominant in SN 1994I at early times while, as demonstrated by Weiler et al. (2007), SN 1993J requires significant contributions to the early absorption from both FFA and SSA. Thus, SN 1994I is the first observed RSN to clearly demonstrate the need for an SSA dominated component to model its radio light curves.

\subsection{External Distant Absorption}

The parameter $K_{4}$ (see Section 3.1) is interpreted as being due to a constant amount of ionized material between us and SN 1994I that is sufficiently far from the SN to be unaffected by the expanding shock wave. It is related to the emission measure (EM) of this intervening ionized hydrogen by Equation (1-223) of Lang (1980) so that

$$
\mathrm{EM}=8.93 \times 10^{7} K_{4}\left(T_{e} / 10^{4} \mathrm{~K}\right)^{1.35} \mathrm{pc} \mathrm{cm}^{-6},
$$

where $T_{e}$ is the electron temperature of the ionized absorbing medium. Thus, the value for $K_{4}$ in Table 4 implies a presence along the line of sight to SN 1994I of ionized hydrogen with an emission measure of $\mathrm{EM} \simeq 4.1 \times 10^{6}\left(T_{e} / 10^{4} \mathrm{~K}\right)^{1.35} \mathrm{pc} \mathrm{cm}^{-6}$.

Similar to the case of SN 1978K (Montes et al. 1997; Chu et al. 1999) this thermal absorption is likely to be due to an intervening $\mathrm{H}$ II region and not part of the matter from the presupernova mass-loss wind. Most $\mathrm{H}$ II regions with an EM higher than $\sim 10^{6} \mathrm{pc} \mathrm{cm}^{-6}$ are known to be rather compact sources (see, e.g., Wilson et al. 1970; Reifenstein et al. 1970); for example, the Orion Nebula has an average EM of $1.8 \times 10^{6} \mathrm{pc} \mathrm{cm}^{-6}$ and a diameter of $\sim 2$ pc (Allen 1973). If we adopt a similar diameter of $\sim 2$ pc for this H II region presumed to be along the line of sight to SN 1994I, we derive an average electron density of $2 \times 10^{3} \mathrm{pc} \mathrm{cm}^{-6}$ and a total mass of the ionized gas of $M(\mathrm{H}$ II $) \simeq$ $250 M_{\odot}$. The volume EM is then $n_{e}^{2} V \simeq 5.1 \times 10^{62} \mathrm{~cm}^{-3}$, which implies a steady source of ionizing radiation of $N_{L} \simeq 1.3 \times 10^{50}$ Lyman-continuum photons $\mathrm{s}^{-1}$. Such a flux is higher than that provided even by a bright O-type star (see, e.g., Panagia 1973) and suggests the presence of a young, compact star cluster in the foreground of SN 1994I.

In order not to make the projection of such a powerful cluster in front of SN 1994I too geometrically improbable, the cluster should be spatially close to the supernova itself; say, separated by no more than $\sim 3 \mathrm{pc}$, which would give a probability of chance projection of $\sim 3 \%$. This then implies that the progenitor of SN 1994I may have been a member of that cluster and, by association, was a massive star.

\subsection{Pre-supernova Mass-loss Rate}

The best-fit value for the parameter $K_{2}$ (see Section 3.1) is related to the density of a presupernova stellar wind. If the density distribution in the CSM around SN 1994I is proportional to $r^{-2}$, as appropriate for a constant mass-loss rate, constant velocity progenitor wind confirmed by Immler et al. (2002), our measurement of $K_{2}=30$ in Table 4 corresponds to a CSM external thermal absorption as a function of time of

$$
\tau(\lambda, t)=0.35 \lambda^{-2.1}(t / 1 \text { day })^{-3} .
$$


Assuming for the moment that the progenitor star for SN 1994I was a red supergiant (RSG), inserting Equation (14) into Equation (16) of Weiler et al. (1986), adopting an electron temperature of $T_{e}=20,000 \mathrm{~K}$ for the absorbing CSM gas, and assuming a wind velocity for this suggested RSG progenitor of $w=10 \mathrm{~km} \mathrm{~s}^{-1}$, we obtain a mass-loss rate of

$$
\begin{aligned}
\dot{M}= & 1.8 \times 10^{-7}\left(v_{s} / 20,000 \mathrm{~km} \mathrm{~s}^{-1}\right)^{1.5}\left(w / 10 \mathrm{~km} \mathrm{~s}^{-1}\right) \\
& \times\left(T_{e} / 20,000 \mathrm{~K}\right)^{0.68} M_{\odot} \mathrm{yr}^{-1} .
\end{aligned}
$$

This estimate of $\dot{M}$ is much lower than the estimate of $1.1 \times$ $10^{-5}\left(w / 10 \mathrm{~km} \mathrm{~s}^{-1}\right) M_{\odot} \mathrm{yr}^{-1}$ obtained by Immler et al. (2002) from their analysis of the SN 1994I X-ray emission data.

Clumping of the CSM (or a systematic deviation from spherical symmetry) might provide a simple explanation for this apparent discrepancy if most of the ionized gas is present in the form of large, isolated structures. In such a case, the massloss rate derived from the X-ray emission, which is integrated over the whole CSM, would be much higher than that estimated from the radio absorption because the latter arises only from the material along the line of sight which may not intercept any dense clumps in the CSM. Indeed, in deriving their $\dot{M}$ estimate Immler et al. (2002) assumed that the CSM was both uniform and spherically symmetric. If such appreciable clumping is present, the average density of the emitting material could be grossly underestimated for radio measurements of absorption along the line of sight by a factor equal to the square root of the clumping factor. The X-ray estimated massloss rate (proportional to the ratio of the X-ray luminosity to the average density) would then be overestimated by the same factor. Conversely, the radio absorption-based estimate would provide only a lower limit to the true mass-loss rate. Thus, with a suitable selection of the clumping factor such a large difference between the radio and X-ray estimates could be accounted for without abandoning the RSG progenitor assumption. For example, if we take a "compromise" value of

$$
\sqrt{\dot{M}(\text { radio }) \times \dot{M}(\mathrm{X}-\text { ray })} \simeq 1.4 \times 10^{-6}\left(w / 10 \mathrm{~km} \mathrm{~s}^{-1}\right) M_{\odot} \mathrm{yr}^{-1}
$$

to be appropriate for SN 1994I progenitor's wind, the clumping factor would be $\sim 40$ and the fraction of mass in the diffuse component $\sim 15 \%$.

On the other hand, if the progenitor of SN 1994I was not an RSG star but a W-R star, as has been suggested for Type Ib/c SNe by many authors (see, e.g., van den Bergh 1988; Gal-Yam et al. 2007; Smartt 2009; and references therein), the W-R progenitor would have a much higher wind speed ( $>10^{3} \mathrm{~km} \mathrm{~s}^{-1}$; see, e.g., Willis 1996), as opposed to $\sim 10 \mathrm{~km} \mathrm{~s}^{-1}$ for an RSG star. Thus, a W-R progenitor would revise the above estimate of the presupernova mass-loss rate from Equation (16) upward to $\dot{M}>1.4 \times 10^{-4} M_{\odot} \mathrm{yr}^{-1}$, a mass-loss rate that is quite possible for a W-R star toward the end of its evolution (see, e.g., Willis 1996).

\section{CONCLUSIONS}

We present detailed radio observations of SN 1994I at multiple wavelengths from a few days to more than eight years after explosion. Although there have been many radio observations of Type Ib/c supernovae since SN 1994I, SN 1994I remains the best-studied and most heavily sampled example of the class. Also, even though observations since those of SN 1994I have indicated that SSA can play a role in the early radio light curves of supernovae that have relatively low density circumstellar matter, such as Type Ib/c and Type IIb, SN 1994I was the first example that was so well sampled early enough that such a conclusion was inescapable. Also, as seen in other RSNe (see Section 4.2) this low-density circumstellar thermal material in SN 1994I may be irregularly distributed as discussed in Section 4.6.

As with previous detailed observations of the radio emission from supernovae, there is no sign of systematic spectral index evolution with time. However, a changing spectral index with frequency, rather than with time, is a distinct possibility, with the highest observed frequencies perhaps indicating a somewhat flatter spectral index.

As has been noted in the few other RSNe which have been sufficiently well studied, the radio light curves, even though their general properties evolve smoothly and are well described by existing models, show significant "bumps and wiggles." SN 1994I shows a significant local "dip" between $\sim 10$ and $\sim 55$ days after explosion that is interpreted as a temporary decrease in the average density of the circumstellar medium, perhaps implying the origin of SN 1994I in an exploding W-R star in a wide binary system with an earlytype stellar companion, although changing mass-loss rate from the progenitor star cannot be ruled out.

The usual scenario for core-collapse (Type Ib/c and Type II) supernovae is that the progenitor was an RSG. However, based on the information available from these new observations, it appears that a W-R star undergoing a period of very high massloss rate is the most likely progenitor star for SN 1994I.

We are deeply indebted to Dr. Schuyler Van Dyk of the Spitzer Science Center for the large amount of work he did on the data collection and editing, and to Dr. Barry Clark of the NRAO for scheduling our numerous observations. Many observers also contributed valuable telescope time to enable such a dense sampling of the radio light curves. K.W.W. thanks the Office of Naval Research (ONR) for the 6.1 funding supporting his research.

\section{REFERENCES}

Allen, C. W. 1973, (3rd ed., London: Univ. London Athlone Press)

Armus, L., \& Mazzarella, J. M. 1994, IAU Circ., 5961

Berger, E., Kulkarni, S. R., Frail, D. A., \& Soderberg, A. M. 2003a, ApJ, 599, 408

Berger, E., Soderberg, A. M., Frail, D. A., \& Kulkarni, S. R. 2003b, ApJ, 587, L5

Chevalier, R. A. 1982a, ApJ, 259, 302

Chevalier, R. A. 1982b, ApJ, 259, L85

Chevalier, R. A. 1998, ApJ, 499, 810

Chu, Y.-H., Caulet, A., Montes, M. J., et al. 1999, ApJ, 512, L51

Clocchiatti, A., Brotherton, M., Harkness, R. P., \& Wheeler, J. C. 1994, IAU Circ., 5972

Feldmeier, J. J., Ciardullo, R., \& Jacoby, G. H. 1997, ApJ, 479, 231

Filippenko, A. V., Barth, A. J., Matheson, T., et al. 1995, ApJ, 450, L11

Filippenko, A., Matheson, T., \& Barth, A. 1994, IAU Circ., 5964

Gal-Yam, A., Leonard, D. C., Fox, D. B., et al. 2007, ApJ, 656, 372

Goss, W. M., Allen, R. J., Ekers, R. D., \& de Bruyn, A. G. 1973, Nature, 243, 42

Gottesman, S. T., Broderick, J. J., Brown, R. L., Balick, B., \& Palmer, P. 1972, ApJ, 174, 383

Immler, S., Pietsch, W., \& Aschenbach, B. 1998, A\&A, 336, L1

Immler, S., Wilson, A. S., \& Terashima, Y. 2002, ApJ, 573, L27

Kellermann, K. I., \& Pauliny-Toth, I. I. K. 1969, ApJ, 155, L71

Kirshner, R. 1994, IAU Circ., 5981

Kulkarni, S. R., Frail, D. A., Wieringa, M. H., et al. 1998, Nature, 395, 663

Lang, K. R. 1980, in Astrophysical Formulae. A Compendium for the Physicist and Astrophysicist, XXIX, 783 (Berlin: Springer), 47 
Lewin, W. H. G., Zimmermann, H.-U., \& Pietsch, W. 1994, IAU Circ., 6019 Li, Z.-Y., \& Chevalier, R. A. 1999, ApJ, 526, 716

Maddox, L. A. 2006, PhD thesis, Univ. Oklahoma (Publication Number: AAT 3222155. Source: DAI-B 67/06, Dec 2006)

Maddox, L. A., Cowan, J. J., Kilgard, R. E., Schinnerer, E., \& Stockdale, C. J. 2007, AJ, 133, 2559

Marcaide, J. M., Martí-Vidal, I., Alberdi, A., et al. 2009, A\&A, 505, 927

Millard, J., Branch, D., Baron, E., et al. 1999, ApJ, 527, 746

Minkowski, R. 1941, PASP, 53, 224

Montes, M. J., Weiler, K. W., \& Panagia, N. 1997, ApJ, 488, 792

Montes, M. J., Weiler, K. W., Van Dyk, S. D., et al. 2000, ApJ, 532, 1124

Montes, M. J., Van Dyk, S. D., Weiler, K. W., Sramek, R. A., \& Panagia, N. 1998, ApJ, 506, 874

Morrison, L. V., \& Argyle, R. W. 1994, IAU Circ., 5999

Natta, A., \& Panagia, N. 1984, ApJ, 287, 228

Osterbrock, D. E. 1974, Astrophysics of Gaseous Nebulae (San Francisco: Freeman), 82

Panagia, N. 1973, AJ, 78, 929

Panagia, N., Sramek, R. A., \& Weiler, K. W. 1986, ApJ, 300, L55

Panagia, N., Van Dyk, S. D., Weiler, K. W., et al. 2006, ApJ, 646, 369

Phillips, M. 1994, IAU Circ., 5966

Phillips, J. A., Chandler, C. J., \& Rupen, M. P. 1994, IAU Circ., 5968

Puckett, T., \& Armstrong, J. 1994, IAU Circ., 5961

Readhead, A. C. S. 1994, ApJ, 426, 51

Reifenstein, E. C., Wilson, T. L., Burke, B. F., Mezger, P. G., \& Altenhoff, W. J. 1970, A\&A, 4, 357

Richmond, M. 1994, IAU Circ., 5966

Richmond, M. W., \& Filippenko, A. V. 1994, IAU Circ., 5961

Richmond, M. W., Filippenko, A. V., Treffers, R. R., \& Van Dyk, S. D. 1994, IAU Circ., 5963

Rodriguez-Pascual, P. M., Monier, R., \& Wamsteker, W. 1994, IAU Circ., 5968

Rudy, R., \& Dotan, Y. 1994, IAU Circ., 5991

Rupen, M. P., Sramek, R. A., Van Dyk, S. D., Weiler, K. W., \& Panagia, N. 1994, IAU Circ., 5963

Ryder, S. D., Sadler, E. M., Subrahmanyan, R., et al. 2004, MNRAS, 349, 1093

Sadler, E. M., Stathakis, R. A., Boyle, B. J., \& Ekers, R. D. 1998, IAU Circ., 6901
Sandage, A., \& Tammann, G. A. 1974, ApJ, 194, 559

Schmidt, B., Challis, P., \& Kirshner, R. 1994, IAU Circ., 5966

Schmidt, B., \& Kirshner, R. 1994, IAU Circ., 5962

Schwarz, D. H., \& Pringle, J. E. 1996, MNRAS, 282, 1018

Smartt, S. J. 2009, ARA\&A, 47, 63

Soderberg, A. M., Chakraborti, S., Pignata, G., et al. 2010, Nature, 463, 513

Soderberg, A. M., Frail, D. A., \& Wieringa, M. H. 2004, ApJ, 607, L13

Soderberg, A. M., Kulkarni, S. R., Berger, E., et al. 2005, ApJ, 621, 908

Soderberg, A. M., Nakar, E., Berger, E., \& Kulkarni, S. R. 2006, ApJ, 638, 930

Sramek, R. A., Panagia, N., \& Weiler, K. W. 1984, ApJ, 285, L59

Sramek, R. A., \& Weiler, K. W. 2003, in Supernovae and Gamma-Ray Bursters, ed. K. W. Weiler (Berlin: Springer), 153

Tongue, T., Graham, M., Stanley, M., Truesdell, L., \& Westphal, D. 1994, IAU Circ., 5990

Tully, R. B. 1988, Nearby Galaxies Catalog (Cambridge: Cambridge Univ. Press)

van den Bergh, S. 1988, ApJ, 327, 156

van Dyk, S. D., Sramek, R. A., Weiler, K. W., \& Panagia, N. 1993, ApJ, 409, 162

Weiler, K. W., Panagia, N., \& Montes, M. J. 2001a, ApJ, 562, 670

Weiler, K. W., Panagia, N., \& Montes, M. J. 2003, in Supernovae and GammaRay Bursters, ed. K. W. Weiler (Berlin: Springer), 367

Weiler, K. W., Panagia, N., Montes, M. J., \& Sramek, R. A. 2002, ARA\&A, 40, 387

Weiler, K. W., Panagia, N., \& Sramek, R. A. 1990, ApJ, 364, 611

Weiler, K. W., Panagia, N., Sramek, R. A., et al. 2001b, in Proc. Space Telescope Science Institute Symp., Supernovae and Gamma-Ray Bursts: The Greatest Explosions Since the Big Bang, ed. M. Livio, N. Panagia, \& K. Sahu (Vol. 13; Cambridge: Cambridge Univ. Press), 198

Weiler, K. W., \& Sramek, R. A. 1980, IAU Circ., 3485

Weiler, K. W., Sramek, R. A., Panagia, N., van der Hulst, J. M., \& Salvati, M. 1986, ApJ, 301, 790

Weiler, K. W., Van Dyk, S. D., Pringle, J., \& Panagia, N. 1992, ApJ, 399, 672

Weiler, K. W., Williams, C. L., Panagia, N., et al. 2007, ApJ, 671, 1959

Willis, A. J. 1996, Ap\&SS, 237, 145

Wilson, T. L., Mezger, P. G., Gardner, F. F., \& Milne, D. K. 1970, A\&A, 6, 364 